\title{
Comment on "Effects of spatial dispersion on electromagnetic surface modes and on modes associated with a gap between two half spaces"
}

\author{
G. L. Klimchitskaya ${ }^{1}$ and V. M. Mostepanenko ${ }^{2}$ \\ ${ }^{1}$ North-West Technical University, Millionnaya St. 5, St.Petersburg, 191065, Russia \\ ${ }^{2}$ Noncommercial Partnership "Scientific Instruments", \\ Tverskaya St. 11, Moscow, 103905, Russia
}

\begin{abstract}
Recently Bo E. Sernelius [Phys. Rev. B 71, 235114 (2005)] investigated the effects of spatial dispersion on the thermal Casimir force between two metal half spaces. He claims that incorporating spatial dispersion results in a negligible contribution from the transverse electric mode at zero frequency as compared to the transverse magnetic mode. We demonstrate that this conclusion is not reliable because, when applied to the Casimir effect, the approximate description of spatial dispersion used is unjustified.
\end{abstract}

PACS numbers: 71.10.-w, 73.25.+i, 71.36.+c, 68.47.De 
Ref. [1] is devoted to the question of how spatial dispersion affects the Casimir force between real metal plates at nonzero temperature. As is mentioned in Ref. [1], there are two quite different predictions in the literature regarding the thermal Casimir force between real metals. According to one prediction [2, 3, 4], at separations of a few hundred nanometers the thermal correction to the Casimir force is negligible and agrees in a qualitative sense with the ideal metal result. At large separations, where the magnitude of the total force is equal to the thermal correction $(z>6 \mu \mathrm{m}$ at $T=300 \mathrm{~K})$, the results for real and ideal metals coincide. According to another prediction [5, 6], at short separations below one micrometer the thermal correction for real metals can be as large as 10-20\% of the total Casimir force. At large separations the thermal force between real metals is one-half of the ideal-metal result. Mathematically the distinction between the two proposed formalisms lies in the fact that in Refs. [2, 3, 4] there is a nonzero contribution from the transverse electric mode at zero frequency, whereas in Refs. [5, 6] this contribution is equal to zero. A nonzero result for the contribution from the transverse electric mode at zero frequency is obtained by using the dielectric function of the plasma model [2, 3] or the Leontovich surface impedance [4]. A zero result follows by application of the Drude dielectric function with a relaxation parameter [5, 6]. As was demonstrated in Refs. [7, 8], the prediction of Refs. [5, 6] is excluded by experiment at $99 \%$ confidence within a wide separation region from 300 to $500 \mathrm{~nm}$, whereas the predictions of Refs. [2, 3, 4] are consistent with experiment. There is also the qualitative disagreement between the prediction of Refs. [5, 6] and the results of the first modern measurement of the Casimir force [9] at $z \approx 1 \mu \mathrm{m}$. At $z=1 \mu \mathrm{m}$ the large thermal correction, predicted in Refs. [5, 6], achieves $19 \%$ of the force in ideal metal case, whereas the experimental uncertainty in [9] was 5-10\%. The predicted thermal effect was not, however, observed in [9]. Ref. [1] claims that the inclusion of spatial dispersion leads to a result which is almost identical to that obtained from the Drude model in Refs. [5, 6]. Below we demonstrate that including spatial dispersion into the theory of the Casimir force as in Ref. [1] is unjustified and, thus, the result obtained in this reference cannot be trusted.

To find the electromagnetic surface modes associated with an empty gap between the two half spaces, Ref. [1] assumes in Secs. III and IV the validity of the standard continuity boundary conditions

$$
E_{1 t}=E_{2 t}, \quad B_{1 n}=B_{2 n}, \quad D_{1 n}=D_{2 n}, \quad B_{1 t}=B_{2 t},
$$


where $\boldsymbol{n}$ is the normal to the boundary directed inside medium, and the subscripts $n, t$ refer to the normal and tangential components, respectively. Here $\boldsymbol{E}$ is the electric field strength, $\boldsymbol{D}$ is the electric displacement, $\boldsymbol{B}$ is the magnetic induction, and only nonmagnetic materials are considered so that for the magnetic field $\boldsymbol{H}=\boldsymbol{B}$ (the subscript 1 refers to a vacuum and subscript 2 to a semispace). To describe the spatial dispersion, Ref. [1] assumes that the longitudinal and transverse dielectric functions depend on both the wave vector and frequency $\varepsilon_{k l}=\varepsilon_{k l}(\boldsymbol{q}, \omega)$. However, in the theory of the thermal Casimir force taking into account spatial dispersion, both assumptions used in Ref. [1], i.e., the boundary conditions (11) and the dielectric permittivities depending on both $\boldsymbol{q}$ and $\omega$, are unjustified.

While on the subject of boundary conditions, we recall that in Casimir-type problems the Maxwell equations of an electromagnetic field in a metal can be written as

$$
\begin{aligned}
& \operatorname{rot} \boldsymbol{E}+\frac{1}{c} \frac{\partial \boldsymbol{B}}{\partial t}=0, \quad \operatorname{div} \boldsymbol{D}=0, \\
& \operatorname{rot} \boldsymbol{B}-\frac{1}{c} \frac{\partial \boldsymbol{D}}{\partial t}=0, \quad \operatorname{div} \boldsymbol{B}=0 .
\end{aligned}
$$

Here it is assumed, that there are no current and charge densities of the external sources (i.e., given independently of $\boldsymbol{E}, \boldsymbol{D}$ and $\boldsymbol{B}$ ). The contribution from the conduction electrons is taken into account by the relation for the determination of $\boldsymbol{D}$

$$
\frac{\partial \boldsymbol{D}}{\partial t}=\frac{\partial \boldsymbol{E}}{\partial t}+4 \pi \boldsymbol{i},
$$

where $\boldsymbol{i}$ is the volume current density induced by the fields $\boldsymbol{E}$ and $\boldsymbol{B}$.

In electrodynamics with spatial dispersion the physical fields $\boldsymbol{E}$ and $\boldsymbol{B}$ at the interface are usually finite, though the displacement $\boldsymbol{D}$ can tend to infinity [10]. Taking this fact into account, and integrating the Maxwell equations (2) over the thickness of the boundary layer (see, e.g., Ref. [11]), one reproduces the first two conditions of Eq. (11), but arrives to modified third and fourth conditions [10, 12]

$$
\begin{aligned}
& E_{1 t}=E_{2 t}, \quad B_{1 n}=B_{2 n}, \\
& D_{2 n}-D_{1 n}=4 \pi \sigma, \quad\left[\boldsymbol{n} \times\left(\boldsymbol{B}_{2}-\boldsymbol{B}_{1}\right)\right]=\frac{4 \pi}{c} \boldsymbol{j} .
\end{aligned}
$$

Here the induced surface charge and current densities are given by

$$
\sigma=\frac{1}{4 \pi} \int_{1}^{2} \operatorname{div}[\boldsymbol{n} \times[\boldsymbol{D} \times \boldsymbol{n}]] d l, \quad \boldsymbol{j}=\frac{1}{4 \pi} \int_{1}^{2} \frac{\partial \boldsymbol{D}}{\partial t} d l .
$$


In linear electrodynamics without spatial dispersion the material equation connecting $\boldsymbol{D}$ and $\boldsymbol{E}$ takes the form

$$
D_{k}(\boldsymbol{r}, t)=\int_{-\infty}^{t} \hat{\varepsilon}_{k l}\left(\boldsymbol{r}, t-t^{\prime}\right) E_{l}\left(\boldsymbol{r}, t^{\prime}\right) d t^{\prime}
$$

(here we consider a medium with time-independent properties). According to Eq. (6) , the electric displacement $\boldsymbol{D}(\boldsymbol{r}, t)$ at a position $\boldsymbol{r}$ and time $t$ is determined by the electric field $\boldsymbol{E}$ at the same point $\boldsymbol{r}$ (the spatial dispersion is absent) but at different times $t^{\prime} \leq t$ (generally speaking, the temporal dispersion is present). It can be easily shown that the substitution of Eq. (6) in Eq. (5) leads to $\sigma=0, \boldsymbol{j}=0$. In other words when only a temporal dispersion is present the boundary conditions (4) coincide with the standard continuity conditions (11). However, it is unjustified to use the boundary conditions (1) in the presence of spatial dispersion as is done in Ref. [1]. Specific examples illustrate that in this case neither $\sigma$ nor $\boldsymbol{j}$ is equal to zero [10, 13]. Note that the boundary conditions (4) are the exact consequence of the Maxwell equations with sharp boundary surfaces and they are obtained for macroscopic physical fields. It is not correct to mix them up with the boundary conditions arising in perturbative theories and for fictitious fields (see below).

We turn now to the second assumption used in Ref. [1], i.e., to the description of spatial dispersion in terms of dielectric functions depending on $\boldsymbol{q}$ and $\omega$. If only a temporal dispersion is present, one can substitute the Fourier transformations

$$
\boldsymbol{E}(\boldsymbol{r}, t)=\int_{-\infty}^{\infty} \boldsymbol{E}(\boldsymbol{r}, \omega) e^{-i \omega t} d \omega, \quad \boldsymbol{D}(\boldsymbol{r}, t)=\int_{-\infty}^{\infty} \boldsymbol{D}(\boldsymbol{r}, \omega) e^{-i \omega t} d \omega
$$

in Eq. (6) and arrive at

$$
D_{k}(\boldsymbol{r}, \omega)=\varepsilon_{k l}(\boldsymbol{r}, \omega) E_{l}(\boldsymbol{r}, \omega),
$$

where

$$
\varepsilon_{k l}(\boldsymbol{r}, \omega)=\int_{0}^{\infty} \hat{\varepsilon}_{k l}(\boldsymbol{r}, \tau) e^{i \omega \tau} d \tau
$$

is the frequency-dependent dielectric permittivity and $\tau \equiv t-t^{\prime}$. Eq. (8) and boundary conditions (11) are used in all traditional derivations of the Lifshitz formula [14, 15, 16, 17, 18, 19, 20] describing the van der Waals and Casimir force between parallel plates (half spaces) possessing only a temporal (in other words "frequency") dispersion.

If the material of the plates possesses both temporal and spatial dispersion, Eq. (6) should be generalized to

$$
D_{k}(\boldsymbol{r}, t)=\int_{-\infty}^{t} d t^{\prime} \int d \boldsymbol{r}^{\prime} \hat{\varepsilon}_{k l}\left(\boldsymbol{r}, \boldsymbol{r}^{\prime}, t-t^{\prime}\right) E_{l}\left(\boldsymbol{r}^{\prime}, t^{\prime}\right)
$$


If the medium were uniform in space (i.e., all points were equivalent), the kernel $\hat{\varepsilon}$ would not depend on $\boldsymbol{r}$ and $\boldsymbol{r}^{\prime}$ separately, but only on the difference $\boldsymbol{R} \equiv \boldsymbol{r}-\boldsymbol{r}^{\prime}$. In this case, by performing the Fourier transformation

$$
\boldsymbol{E}(\boldsymbol{r}, t)=\int_{-\infty}^{\infty} d \omega \int d \boldsymbol{q} \boldsymbol{E}(\boldsymbol{q}, \omega) e^{i(\boldsymbol{q} r-\omega t)}
$$

(and the same for $\boldsymbol{D}$ ), and substituting it in Eq. (10), one could introduce the dielectric permittivities

$$
\varepsilon_{k l}(\boldsymbol{q}, \omega)=\int_{0}^{\infty} d \tau \int d \boldsymbol{R} \hat{\varepsilon}_{k l}(\boldsymbol{R}, \tau) e^{-i(\boldsymbol{q} \boldsymbol{R}-\omega \tau)} .
$$

These permittivities depend on both the wave vector and frequency and bring Eq. (10) into the form

$$
D_{k}(\boldsymbol{q}, \omega)=\varepsilon_{k l}(\boldsymbol{q}, \omega) E_{l}(\boldsymbol{q}, \omega)
$$

in analogy to Eq. (8) .

In Ref. [1], however, the system under consideration is not uniform due to the presence of a macroscopic gap between the two half spaces. Because of this, it is impossible to assume that the kernel $\hat{\varepsilon}$ depends only on $\boldsymbol{R}$ and $\tau$, and hence it is not possible to introduce $\varepsilon_{k l}(\boldsymbol{q}, \omega)$. In fact in the presence of boundaries the kernel $\hat{\varepsilon}$ for systems with spatial dispersion depends not only on $\boldsymbol{R}$ and $\tau$ but also on the distance from the boundary [10]. An approximate phenomenological approach to dealing with this situation is described in Ref. [10]. For electromagnetic waves with a wavelength $\lambda$ the kernel $\hat{\varepsilon}\left(\boldsymbol{r}, \boldsymbol{r}^{\prime}, \tau\right)$ is significantly large only in a certain vicinity of the point $\boldsymbol{r}$ with characteristic dimensions $a \ll \lambda$ (in fact for nonmetallic condensed media $a$ is of the order of the lattice constant). One can then assume that $\hat{\varepsilon}$ is a function of $\boldsymbol{R}=\boldsymbol{r}-\boldsymbol{r}^{\prime}$, except for a layer of thickness $a$ adjacent to the boundary surface. If one is not interested in this subsurface layer, the quantity $\varepsilon_{k l}(\boldsymbol{q}, \omega)$ may be used to describe the remainder of the medium.

This approximate phenomenological approach is widely applied in the theory of the anomalous skin effect (see, e.g., Ref. [21]) for the investigation of bulk physical phenomena involving electromagnetic fields. In Ref. [21] some kind of fictitious infinite system is introduced and the electromagnetic fields in this system are discontinuous on the surface. This discontinuity should not be confused with the discontinuity of physical fields of a real system in the presence of spatial dispersion given by Eqs. (4) and (5). (There is, however, another approach which describes the anomalous skin effect in polycrystals in terms of the 
local Leontovich impedance taking into account the shape of Fermi surface [22]). The frequency and wave vector dependent dielectric permittivity in the presence of boundaries is also successfully applied in some other problems, e.g., in the theory of radiative heat transfer [23] or in the study of electromagnetic interactions of molecules with metal surfaces [24]. All these applications are in more or less good agreement with experiment. The boundary effects can be taken into account by the boundary conditions (44) supplemented in some way by so called "additional boundary conditions".

It is unlikely, however, that the phenomenological approach of Ref. [21] using the frequency and wave vector dependent dielectric permittivity would be applicable for the calculation of Casimir force between metal surfaces, where the boundary effects for vacuum electromagnetic oscillations are of prime importance and contribute significantly to the total result. Ref. [1] uses the quantities $\varepsilon_{k l}(\boldsymbol{q}, \omega)$ in a spatially nonuniform system of two half spaces separated by a gap without justification, and determines the surface modes from the continuity conditions (1) instead of the boundary conditions (44) valid in the presence of spatial dispersion. As was emphasized in Ref. [10], in the case of a bounded medium it is inadmissible to use the same kernel in Eq. (10) which is appropriate for an unbounded medium. According to Ref. 25], this leads to a violation of the law of conservation of energy. It is then not surprising that in doing so Ref. [1] arrives to an incorrect contribution from the transverse electric mode to the Casimir free energy. However, in addition to Ref. [1] some other recent publications (see, e.g., Refs. [26, 27]) apply the dielectric permittivities $\varepsilon_{k l}(\boldsymbol{q}, \omega)$ obtained for an unbounded medium to the theory of the Casimir effect which essentially depends on the presence of boundaries.

One more shortcoming of Ref. [1] is that it uses the usual Lifshitz formula for the free energy derived in the presence of only temporal dispersion [14, 15, 16, 17, 18, 19, 20], to compute the effects of spatial dispersion. It has been known, however, that with the inclusion of spatial dispersion the free energy of a fluctuating field takes the form $\mathcal{F}=\mathcal{F}_{L}+\Delta \mathcal{F}$, where $\mathcal{F}_{L}$ is given by the Lifshitz formula derived in a spatially local case and written in terms of the Fresnel reflection coefficients, and $\Delta \mathcal{F}$ is an additional term which can be expressed in terms of the thermal Green's function of electromagnetic field and polarization operator [28]. The review paper [28] contains a few references to incorrect results by different authors obtained by the substitution of $\varepsilon_{k l}(\boldsymbol{q}, \omega)$, taking account of spatial dispersion, into the usual Lifshitz formula. During the last years several more similar papers have been published 
(see, e.g., Refs. [26, 27]). It can be true that the Lifshitz formula written in terms of general reflection coefficients is applicable in both spatially local and nonlocal situations. However, as far as the exact reflection coefficients in a spatially nonlocal case are unknown, the use of some approximate models, elaborated in the literature for applications different than the Casimir effect, may lead to incorrect results for $\Delta \mathcal{F}$ and create serious inconsistencies with experiment.

To conclude, the main purpose of Ref. [1] "to find out how spatial dispersion affects the Casimir force between real metal plates" is in our opinion not achieved. Instead of using the boundary conditions (44) valid in the presence of spatial dispersion, and the appropriate generalization of the Lifshitz formula, Ref. [1] uses the continuity conditions (11) and usual Lifshitz formula derived in the presence of only temporal dispersion. Ref. [1] also applies the phenomenological dielectric permittivity which depends on frequency and wave vector to the calculation of the Casimir force, a phenomenon primarily determined by virtual photons. These unjustified assumptions are responsible for the main conclusion of Ref. [1] that spatial dispersion results in practically the same "dramatic effect" that was previously obtained in Ref. [5] from the Drude dielectric function: the contribution from the transverse electric mode at zero frequency "completely vanishes if dissipation is included", and is negligibly small compared to the contribution from the transverse magnetic mode if dissipation is absent. Ref. [1] does not inform the reader that theoretical approaches with zero or negligibly small contribution from the transverse electric mode at zero frequency have already been excluded experimentally at 99\% confidence in Refs. [7, 8]. By now such approaches are rejected by 4 different experiments [29] including the experiment of Ref. [9]. At the same time, the Leontovich impedance approach, which leads to a nonzero contribution from the transverse electric mode at zero frequency, is both consistent with experiment and takes dissipation into account [4, 7, 8, 30]. The physical reasons why the Leontovich impedance approach is to be preferred are articulated in Ref. [30].

As to the role of spatial dispersion in the Casimir effect, it was first investigated using the Leontovich surface impedance in Ref. [31]. The Leontovich approach can be considered as an approximate phenomenological approach. As was shown above, the approach of Ref [1], in spite of the author's claims to the contrary, is also phenomenological and approximative. At the moment there is no fundamental theory which describes the thermal Casimir force between real metals incorporating spatial nonlocality. Because of this, one should choose 
between different approximate aproaches, and in so doing the role of experiment must not be underestimated. It is well understood that at laboratory temperatures of about $300 \mathrm{~K}$ and at Casimir plate separations of a few hundred nanometers (the frequency region of infrared optics) spatial dispersion does not play any role. It can be confidently neglected also in the region of the normal skin effect, i.e., at separations between the plates greater than $4-5 \mu \mathrm{m}$. If some approximate phenomenological approach leads to the opposite conclusion, there are strong reasons to believe that it is not reliable.

\section{Acknowledgments}

This work was supported by the Russian Foundation for Basic Research (Grant No. 05-08-18119a).

[1] B. E. Sernelius, Phys. Rev. B 71, 235114 (2005).

[2] C. Genet, A. Lambrecht, and S. Reynaud, Phys. Rev. A 62, 012110 (2000).

[3] M. Bordag, B. Geyer, G. L. Klimchitskaya, and V. M. Mostepanenko, Phys. Rev. Lett. 85, $503(2000)$.

[4] B. Geyer, G. L. Klimchitskaya, and V. M. Mostepanenko, Phys. Rev. A 67, 062102 (2003).

[5] M. Boström and B. E. Sernelius, Phys. Rev. Lett. 84, 4757 (2000).

[6] J. S. Høye, I. Brevik, J. B. Aarseth, and K. A. Milton, Phys. Rev. E 67, 056116 (2003).

[7] R. S. Decca, E. Fischbach, G. L. Klimchitskaya, D. E. Krause, D. López, and V. M. Mostepanenko, Phys. Rev. D 68, 116003 (2003).

[8] R. S. Decca, D. López, E. Fischbach, G. L. Klimchitskaya, D. E. Krause, and V. M. Mostepanenko, Ann. Phys. (N.Y.) 318, 37 (2005).

[9] S. K. Lamoreaux, Phys. Rev. Lett. 78, 5 (1997); 81, 5475 (1998).

[10] V. M. Agranovich and V. L. Ginzburg, Crystal Optics with Spatial Dispersion, and Excitons (Springer, Berlin, 1984).

[11] J. A. Stratton, Electromagnetic Theory (McGraw-Hill, New York, 1941).

[12] V. L. Ginzburg, Physics and Astrophysics (Pergamon Press, Oxford, 1985).

[13] V. M. Agranovich and V. L. Ginzburg, Zh. Eksp. Teor. Fiz. 63, 838 (1972) [Sov. Phys. JETP 
36, $440(1973)]$.

[14] I. E. Dzyaloshinskii, E. M. Lifshitz, and L. P. Pitaevskii, Usp. Fiz. Nauk 73, 381 (1961) [Sov. Phys. Usp. (USA) 4, 153 (1961)].

[15] E. M. Lifshitz and L. P. Pitaevskii, Statistical Physics, Part 2 (Pergamon Press, Oxford, 1980).

[16] P. W. Milonni, The Quantum Vacuum ( Academic Press, San Diego, 1994).

[17] V. M. Mostepanenko and N. N. Trunov, The Casimir Effect and Its Applications (Clarendon Press, Oxford, 1997).

[18] M. Schaden and L. Spruch, Phys. Rev. A 58, 935 (1998).

[19] G. L. Klimchitskaya, U. Mohideen, and V. M. Mostepanenko, Phys. Rev. A 61, 062107 (2000).

[20] M. Bordag, U. Mohideen, and V. M. Mostepanenko, Phys. Rep. 353, 1 (2001).

[21] K. L. Kliewer and R. Fuchs, Phys. Rev. 172, 607 (1968).

[22] I. M. Kaganova and M. I. Kaganov, Phys. Rev. B 63, 054202 (2001).

[23] S. M. Rytov, Yu. A. Kravtsov, and V. I. Tatarskii, Principles of Statistical Radiophysics, Vol.3 (Springer-Verlag, Berlin, 1989).

[24] G. W. Ford and W. H. Weber, Phys. Rep. 113, 195 (1984).

[25] J. T. Foley and A. J. Devaney, Phys. Rev. B 12, 3104 (1975).

[26] V. B. Svetovoy and R. Esquivel, Phys. Rev. E 72, 036113 (2005); R. Esquivel and V. B. Svetovoy, Phys. Rev. A 69, 062102 (2004).

[27] R. Esquivel, C. Villarreal, and W. L. Mochán, Phys. Rev. A 68, 052103 (2003); A. M. Contreras-Reyes and W. L. Mochán, Phys. Rev. A 72, 034102 (2005).

[28] Yu. S. Barash and V. L. Ginzburg, Usp. Fiz. Nauk 116, 5 (1975) [Sov. Phys. Usp. (USA) 18, 305 (1975)].

[29] V. B. Bezerra, R. S. Decca, E. Fischbach, G. L. Klimchitskaya, D. E. Krause, D. López, V. M. Mostepanenko, and C. Romero, Phys. Rev. E 73, 028101 (2006).

[30] S. K. Lamoreaux, Rep. Progr. Phys. 68, 201 (2005); J. R. Torgerson and S. K. Lamoreaux, Phys. Rev. E 70, 047102 (2004).

[31] E. I. Kats, Zh. Eksp. Teor. Fiz. 73, 212 (1977) [Sov. Phys. JETP 46, 109 (1977)]. 